\title{
Invited Talk: Big Data in Medical Image Computing
}

\author{
Wiro Niessen \\ Erasmus MC Rotterdam, The Netherlands \\ Delft University of Technology, Delft, The Netherlands \\ Quantib BV, Rotterdam, The Netherlands \\ w.niessen@erasmusmc.nl
}

Big data are dramatically increasing the possibilities for prevention, cure and care, and changing the landscape of the healthcare system. Will artificial intelligence make doctors obsolete or give them more possibilities? Will citizens be delivered into the hands of anonymous information systems or will they gain more control over their personal health. It is difficult to predict the speed of change and impact of both big data and artificial intelligence on health care, but it is clear that changes will be tremendous.

In this presentation, I will show examples of possible large benefits. For example how large scale data analytics in longitudinal population neuroimaging studies, especially when combining imaging with other clinical, biomedical and genetic data, provides a unique angle to study the brain, both in normal ageing and disease. And how this can be the basis of new methods for disease detection, diagnosis, and prognosis in clinical practice. 